\title{
Characterization of N-polar GaN/AIGaN/GaN Heterostructures Using Electron Holography
}

\author{
A. Boley, ${ }^{1}$ D. F. Storm, ${ }^{2}$ M.R. McCartney ${ }^{1}$, and D. J. Smith ${ }^{1}$ \\ ${ }^{1}$ Department of Physics, Arizona State University, Tempe, AZ 85287 \\ ${ }^{2}$ Naval Research Laboratory, Washington, DC 20375
}

III-nitride high-electron mobility transistors (HEMTs) are in demand as commercial power amplifying devices based on their high breakdown field and high operating voltage, as well as wide band gap [1]. As opposed to the standard Ga-polar heterostructures, N-polar devices are better suited for sensors and enhancement mode transistors [2]; advantages include a strong back-barrier and low contact resistance [3]. One important feature of nitride-based HEMTs is the occurrence of a two-dimensional electron gas (2DEG) at or near the interface between adjacent layers [4]. The location of the 2DEG in nitride HEMTs has been shown to vary depending on whether the material is Ga-polar or N-polar. The 2DEG in GaN/AlGaN/GaN heterostructures grown on sapphire is located just above the upper GaN/AlGaN interface for the N-polar structure, and just below the lower $\mathrm{AlGaN} / \mathrm{GaN}$ interface for the conventional Ga-polar structure [5]. In the current work, N-polar GaN/A10.3Ga0.7N/GaN HEMT structures were grown on GaN substrates, as shown in Fig. 1, samples for TEM observation were prepared using Focused Ion Beam (FIB) standard lift-out and backside milling techniques (FEI Nova 200), and the piezoelectric fields and sheet charge were investigated using off-axis electron holography (Phillips CM200 FEG).

Electron holography was performed, as shown in Fig. 2, and phase (Fig. 3(a)), amplitude, and thickness (Fig. 3(b)) images were reconstructed. A mean free path of $70 \mathrm{~nm}$ was used for $\mathrm{GaN}$ in the calculation of thickness images, so the apparent thickness is different in the AlGaN layer because of its different mean free path. A profile of the phase image in Fig. 3(a) revealed a slope in the lower GaN layer due to changing sample thickness. This slope was subtracted from the profile, and the linear slope across the AlGaN layer in the resulting phase profile, shown in Fig. 4, confirms the presence of the electric field. Potential profiles were calculated from phase profiles using $\Delta V=\Delta \varphi /\left(\mathrm{C}_{E}{ }^{*} \mathrm{t}\right)$, where $\Delta V$ is the change in potential, $\Delta \varphi$ is the phase shift of the electrons in the sample, $C_{E}$ is a constant that depends on the energy of the incident electron, and $t$ is the thickness of the sample [6]. The thickness used was the value in the lower GaN layer next to the AlGaN, and is $120 \mathrm{~nm}$ for the profile shown in Fig. 4. The field was calculated by fitting the slope across the AlGaN layer of the potential profiles of several electron holograms: preliminary results give a value of $E=0.12 \pm 0.02 \mathrm{MV} / \mathrm{cm}$. The $2 \mathrm{DEG}$ is expected to be near the upper GaN/AlGaN interface, and next steps include higher resolution electron holography to locate and quantify this $2 \mathrm{DEG}$.

[1] U.K. Mishra, et al., Proc. IEEE 90 (2002) 1022.

[2] S. Rajan, et al., J. Appl. Phys. 102 (2007) 044501.

[3] M.H. Wong, et al., Semicond. Sci. Technol. 28 (2013) 074009.

[4] D.J. Smith, et al., Microelectron. Reliab. 50 (2010) 1514.

[5] R. Dimitrov, et al., J. Appl. Phys. 87 (2000) 375.

[6] M.R. McCartney and D.J. Smith, Annu. Rev. Mater. Res. 37 (2007) 729.

[7] The recent work at ASU was supported under contract to Wyle Laboratories as part of RIAC Contract

HC1047-05-D-4005. We gratefully acknowledge the use of facilities within the John M. Cowley Center for High

Resolution Electron Microscopy at Arizona State University. 


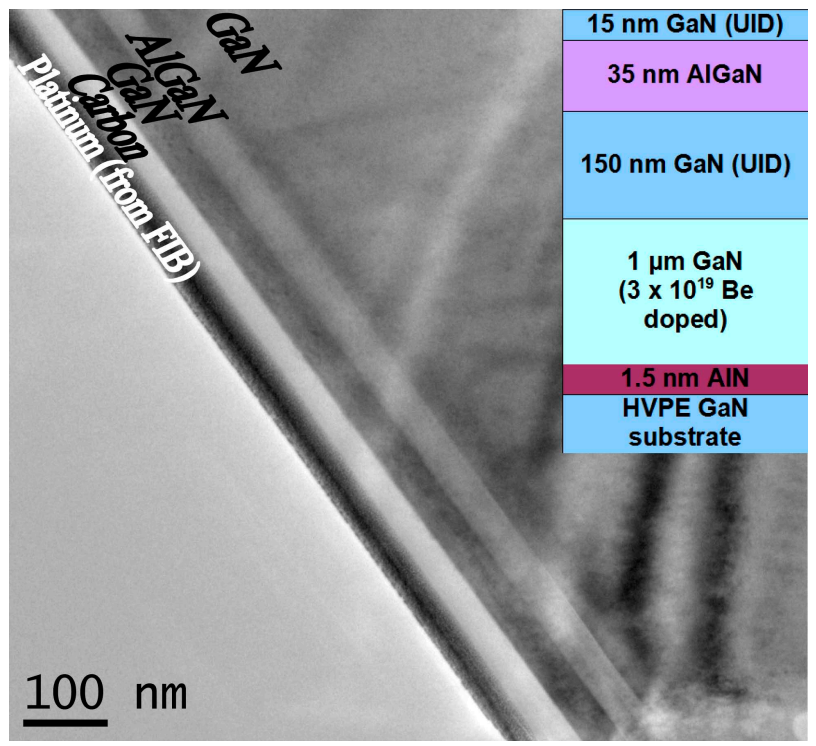

Fig. 1. XTEM image showing $\mathrm{GaN} / \mathrm{AlGaN} / \mathrm{GaN}$ heterostructure, with nominal structure inset.

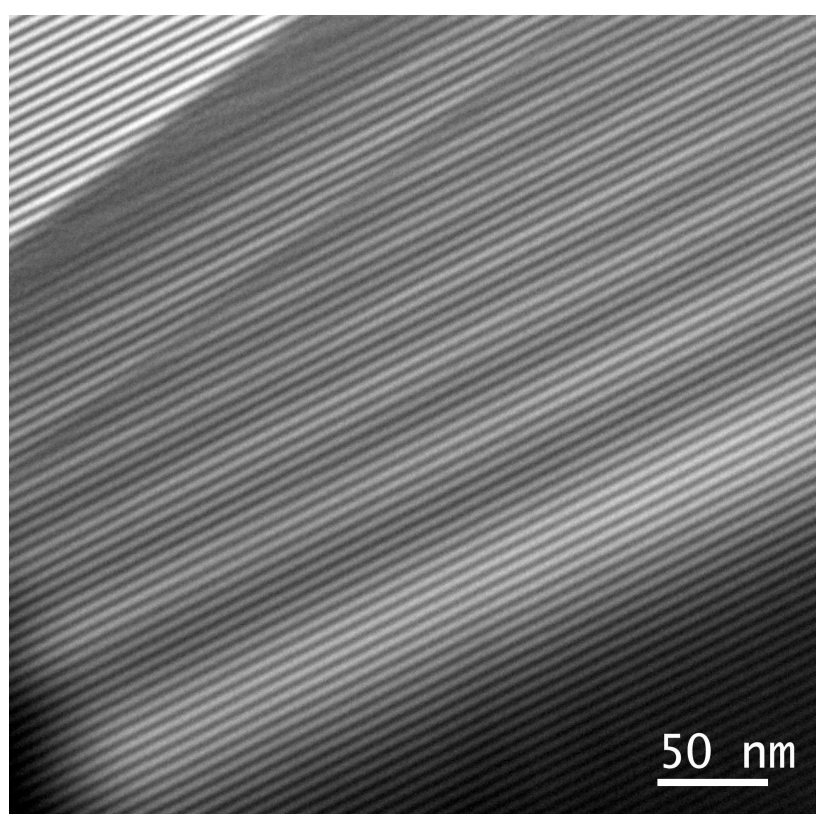

Fig. 2. Electron hologram of $\mathrm{GaN} / \mathrm{AlGaN} / \mathrm{GaN}$ heterostructure.

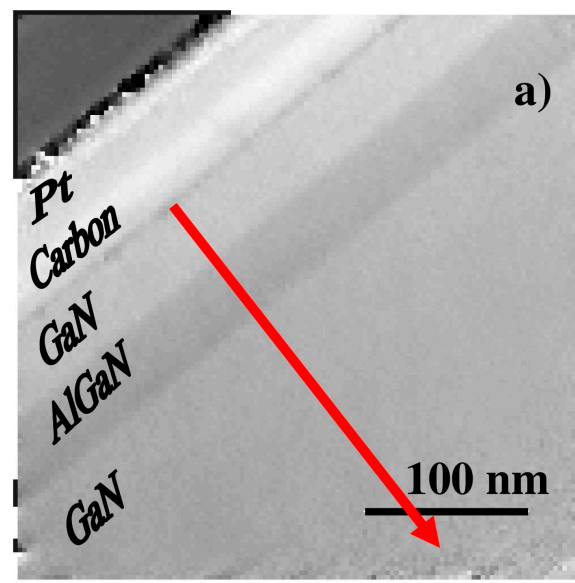

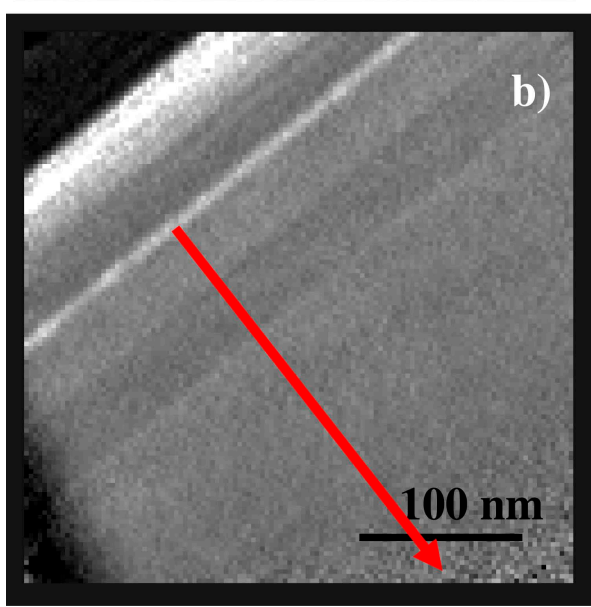

Fig. 3. (a) Phase and (b) thickness images reconstructed from the hologram in Fig. 2.

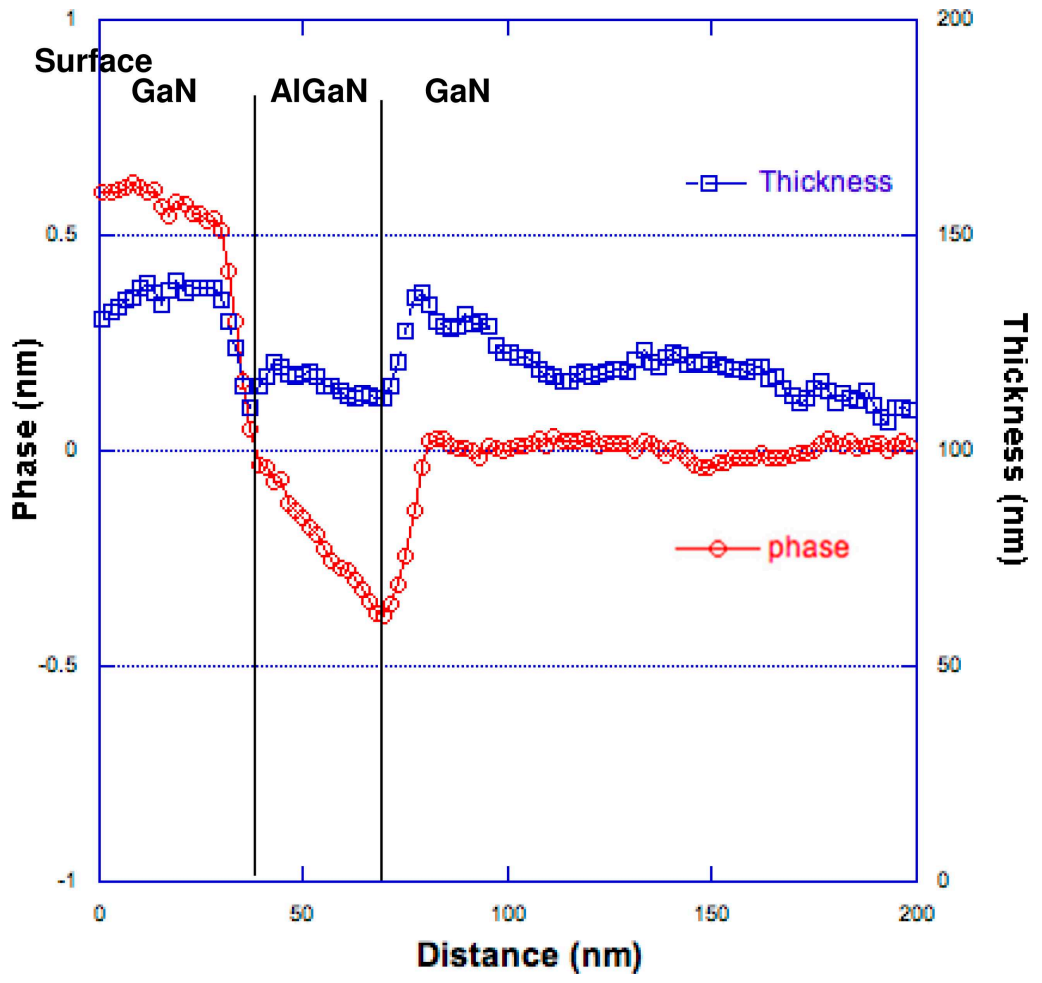

Fig. 4. Thickness profile and flattened phase profile from Figs. 3(b) and (a), respectively. The profile width used for averaging was approximately $90 \mathrm{~nm}$. 